\title{
The Danger of Being Too Sympathetic: Norepinephrine in Alzheimer's Disease and Graying of Hair
}

\author{
Andrew R. Mendelsohn ${ }^{1,2}$ and James W. Larrick ${ }^{1,2}$
}

\begin{abstract}
Although alterations in the sympathetic nervous system (SNS) with age have been reported, and serious degenerative diseases of the autonomic nervous system such as multiple system atrophy are more likely to strike older people, connections between dysregulated adrenergic receptors and age-associated diseases and phenotypes have not been well studied. Two recent reports suggest that SNS may be more closely connected than previously appreciated. First, low nanomolar concentrations of Alzheimer's disease (AD)-associated A $\beta 42$-amyloid oligomers alter signaling by SNS neurotransmitter norepinephrine (NE) to sufficiently activate kinase GSK3 $\beta$ to hyperphosphorylate tau, a key mediator of neurotoxicity in AD. Connecting beta-amyloid to tau in AD has been a key quest in understanding $\mathrm{AD}$ and developing therapeutics. The $\alpha_{2}$ adrenergic receptor inhibitory drug idazoxan reduces GSK $3 \beta$ activity and tau phosphorylation in AD mice with improved cognitive function, even in the presence of betaamyloid deposits. In this study, SNS activation in the brain coupled with problematic A $\beta 42$-amyloid oligomers result in serious consequences that can be ameliorated by reducing SNS signaling. A second example of the detrimental effects of increased SNS signaling is the premature graying of hair in response to stress. Secretion of NE resulting from stress causes differentiation of most hair pigment melanocyte stem cells (MeSCs) into melanocytes, rapidly depleting the hair follicle of pigment-producing cells as mature melanocytes undergo apoptosis and MeSCs are eventually eliminated. Blockade of NE SNS signaling preserves hair coloration in stressed animals. Increased SNS activation has serious apparently irreversible effects on homeostasis in both situations. Although neither report directly addresses aging, given that $\mathrm{AD}$ and the loss of hair pigmentation have strong age associations, it is of interest to better understand the role that SNS has in promoting age-associated phenotypes generally and determine if tuning the SNS through drug-mediated attenuation of SNS signaling may be of medical benefit.
\end{abstract}

Keywords: sympathetic nervous system, tau, beta-amyloid, gray hair, Alzheimer's disease

\section{Introduction}

$\mathbf{T}$ HE AUTONOMIC NERVOUS SYSTEM (ANS) comprises at least two components, the sympathetic nervous system (SNS) that generally regulates fight, flight or freeze responses and the parasympathetic nervous system that regulates "rest or digest" responses, generally but not always antagonized by the SNS to maintain organismal homeostasis.

Norepinephrine (NE), aka noradrenaline, is a key neurotransmitter of the SNS, being released hormonally from the adrenals as a systemic regulator and locally through SNS neurons.

Tonic central nervous system sympathetic nerve firing helps maintain resting vasomotor tone. ${ }^{1}$ The SNS is involved in regulation of renal functions, metabolism, and blood pressure through altering the arterial baroreflex. ${ }^{2}$ Chronic SNS activity can cause pathological conditions such as hypertension, ${ }^{3}$ renal failure, ${ }^{4}$ endothelial dysfunction, ${ }^{5}$ diastolic dysfunction, ${ }^{6}$ increases in ventricular and aortic wall thickness, ${ }^{7}$ and metabolic abnormalities. ${ }^{8}$ Moreover, there is a tendency for only specific target organs to be affected by changes in SNS activation to have a "sympathetic signature" depending on its source: heart failure leads to increased kidney SNS activity, but not in the gut. ${ }^{1}$

Aging as well as obesity has been known to alter the SNS, especially through increased activation observed in cardiovascular diseases such as hypertension and heart failure. It has been speculated that cell senescence may be a key factor linked to activation of the SNS, although specifics remain to be elucidated. ${ }^{1}$ Moreover, disruption of circadian peripheral clock with age has its basis in SNS dysfunction, which in turn may be due to decline of sympathetic-adrenal-medullary and hypothalamic-pituitary-adrenal axes. ${ }^{9}$ The former potentially

\footnotetext{
${ }^{1}$ Regenerative Sciences Institute, Sunnyvale, California
}

${ }^{2}$ Panorama Research Institute, Sunnyvale, California. 
connects SNS dysfunction to the hypothalamus, ${ }^{10}$ which has been hypothesized by some to exert control over the rate of aging in mammals. ${ }^{11}$

In fact, the idea that old age represents a "hyperadrenergic state" was noted and investigated over 40 years ago. ${ }^{12} \mathrm{Ca}-$ techolamines, epinephrine, and norepinephrine are critical mediators of daily homeostasis as well as the episodic "flight or fight" response. Twenty years ago, Milne and Hong hypothesized that beta receptor blockage pharmacotherapy might increase longevity by decreasing sympathetic "stress." 13 Before proceeding, it's useful to understand that SNS occurs through these two hormones/neurotransmitters targeting adrenergic receptors which include $\alpha 1 \mathrm{~A}, \alpha 1 \mathrm{~B}, \alpha 1 \mathrm{D}, \alpha 2 \mathrm{~A}, \alpha 2 \mathrm{~B}, \alpha 2 \mathrm{C}, \beta 1, \beta 2$ and $\beta 3$ receptors. Given the association of SNS activation with aging, perhaps it's not surprising that $\beta 1$-adrenergic inhibitors, metoprolol and nebivolol modestly increase lifespan in both Drosophila and mice. ${ }^{14}$ Beta blockers are typically prescribed to lower heart rate and blood pressure, which raises the possibility that increased lifespan is due to effects on heart rate. There is a close inverse relationship between the heart rate and lifespan across all species evaluated including humans. ${ }^{15,16}$ Numerous studies have shown an elevated heart rate to be an independent risk factor for cardiovascular disease. ${ }^{17,18,19,20}$ Furthermore, reduced heart rate is associated with reduced cardiovascular mortality. ${ }^{21,22,18,23}$ Thus, the question arises, can artificially lowering heart rate itself have a benefit to organismal longevity? In fact, Gent et al. addressed this question directly by treating mice with ivabradine, ${ }^{24,25}$ a selective inhibitor of the pacemaker current in sinoatrial tissue resulting in a decrease in the diastolic depolarization and consequently heart rate. ${ }^{26} \mathrm{C} 57 \mathrm{BL} 6 / \mathrm{J}$ mice receiving lifelong $50 \mathrm{mg}$ ivabradine/kg/day in drinking water exhibited a $14 \%$ reduction in heart rate with a $6.2 \%$ in median lifespan, similar to the $10 \%$ lifespan increase observed by Spindler et al. for metoprolol. Ivabradine has received EMA and FDA approval for systolic heart failure and chronic stable angina without significant adverse effects. Whether ivabradine or SNS modulator metoprolol would have the same beneficial effects in humans is an open question worth considering. A more general question is where else would attenuating SNS signaling be beneficial? A question worth considering.

There are a large set of pathologies and phenotypic changes associated with human aging. Of these, Alzheimer's disease (AD) is probably one of the most significant, as it involves irreversible neurodegenerative changes in the brain and may afflict as many as $9.7 \%$ of people in the United States $>75$ years, with risk increasing with each subsequent year. As many as $40.7 \%$ of centenarians will have AD. ${ }^{27} \mathrm{AD}$ neurodegeneration has been thought to result from a complex pathology that includes modifications to beta-amyloid and tau, with a synergistic interaction between the two hypothesized to play a key role in driving $\mathrm{AD}$ pathology. However, the mechanisms linking these two key players remain obscure. ${ }^{28}$ Recent study suggests that the SNS may play a role. ${ }^{29}$

\section{Increased SNS Signaling by NE Connects Beta-Amyloid Oligomers to Tau Hyperphosphorylation Resulting in Pathology of AD}

In one of several recent reports that tie beta-amyloid to toxic alterations in microtubule associated protein tau, Zhang et al. describe a novel and potentially important mechanism that contributes to the development of AD. ${ }^{29}$

Zhang et al. focus on the $\alpha_{2 A}$ adrenergic receptor $\left(\alpha_{2 A} A R\right)$ a G protein-coupled receptor, which is present in both noradrenergic and non-noradrenergic receptors in the brain, especially the locus coeruleus, which contain numerous noradrenergic receptors, and is an early target for neurodegeneration. First, experiments on postmortem prefrontal cortexes demonstrated increased $\alpha_{2 \mathrm{~A}} \mathrm{AR}$ signaling activity in brains from $\mathrm{AD}$ patients. This led to checking a database of Alzheimer's patients to determine if blood pressure drug clonidine, a known activator of $\alpha_{2 \mathrm{~A}} \mathrm{AR}$ had effects on cognition, which it did, but only in $\mathrm{AD}$ patients consistent with the possibility that activating $\alpha_{2 \mathrm{~A}}$ AR was detrimental in AD. ${ }^{29}$

To explore this further, Zhang et al. used two different transgenic mouse models: one carrying two human dominant mutations that cause AD in humans and neuropathology in mice: a mutation in the APP gene, which encodes the precursor protein to beta-amyloid, and a mutation in the PS1 gene, which encodes presenilin, a protease that converts amyloid precursor to beta-amyloid. The second mouse model carrying related mutation of APP integrated to express more physiological levels of APP. The mutant mice at 7 months accumulate significant beta-amyloid. They possessed neurons with $\alpha_{2 \mathrm{~A}} \mathrm{AR}$ that were more sensitive to their ligand $\mathrm{NE}$ ex vivo and to the sedation response induced by another $\alpha_{2 \mathrm{~A}} \mathrm{AR}$ activator in vivo. Importantly, in very young 5 -week-old mice, before accumulation of A $\beta 42$ oligomers $\left(\mathrm{A} \beta_{\mathrm{O}}\right)$, thought to be the active pathological form of betaamyloid, there was no difference in $\alpha_{2 \mathrm{~A}} \mathrm{AR}$ between mutant mice and wild-type negative controls. This led to the idea that $\mathrm{A} \beta_{\mathrm{O}}$ is involved in $\alpha_{2 \mathrm{~A}} \mathrm{AR}$ activation, possibly by direct receptor binding.

To test this idea, $\alpha_{2 \mathrm{~A}} \mathrm{AR}$ was expressed in human HEK293 cells in culture. Flow cytometry using a fluorescent antibody to detect $\mathrm{A} \beta_{\mathrm{O}}$ showed that cells expressing $\alpha_{2 \mathrm{~A}} \mathrm{AR}$ bound $\mathrm{A} \beta_{\mathrm{O}}$ at high affinity. Related receptors such as $\alpha_{2 \mathrm{C}} \mathrm{AR}$ were not bound in similar experiments. Computational modeling suggested that $\mathrm{A} \beta_{\mathrm{O}}$ likely bound $\alpha_{2 \mathrm{~A}} \mathrm{AR}$ in the third extracellular loop, which was borne out by mutation studies on this region. This region is distinct from the $\mathrm{NE}$ binding domain, so Zhang et al. hypothesized that $\mathrm{A} \beta_{\mathrm{O}}$ is an allosteric regulator of $\alpha_{2 \mathrm{~A}} \mathrm{AR}$. Indeed they found that $\mathrm{A} \beta_{\mathrm{O}}$ enhances $\mathrm{NE}$ binding and vice versa $\mathrm{NE}$ enhances $\mathrm{A} \beta_{\mathrm{O}}$ binding. Essentially these are positive allosteric regulators of each other. These data were confirmed in vivo using a strain of mice in which an immuno-tagged $\alpha_{2 \mathrm{~A}} \mathrm{AR}$ was co-expressed in individuals carrying $\mathrm{AD}$-associated APP mutations. The interaction between $\mathrm{A} \beta_{\mathrm{O}}$ and $\alpha_{2 \mathrm{~A}} \mathrm{AR}$ was shown in an experiment in which the tagged $\alpha_{2 \mathrm{~A}} \mathrm{AR}$ is bound to a solid matrix and then the binding of associated $\mathrm{A} \beta_{\mathrm{O}}$ is assessed on a Western blot.

Zhang et al. then made a leap to connect the altered function of $\alpha_{2 \mathrm{~A}} \mathrm{AR}$ to other known players in the pathogenesis of AD. Using a panel of protein kinases, they found that GSK $3 \beta$ was activated by dephosphorylation on serine 9 . Not only that but also adding $\alpha_{2 \mathrm{~A}} \mathrm{AR}$ activator clonidine required 100 -fold less $\mathrm{A} \beta_{\mathrm{O}}$ than was previously reported to activate GSK3 $\beta$. Downstream tau, another key AD player, became hyperphosphorylated, a potentially cytotoxic change. A 100-fold enhancement of response sensitivity of GSK $3 \beta /$ phospho tau formation to $\mathrm{A} \beta_{\mathrm{O}}$ is potentially of great significance. 
Cells carrying a mutant $\alpha_{2 \mathrm{~A}} \mathrm{AR}$ that could not bind $\mathrm{A} \beta_{\mathrm{O}}$ did not demonstrate similar tau hyperphosphorylation. These results were also confirmed in vivo. The dorsal hippocampus of normal mice, which have physiological levels of $\mathrm{NE}$, were microinjected with $\mathrm{A} \beta_{\mathrm{O}}$ and either saline, lithium, a GSK $3 \beta$ inhibitor, or idazoxan, an $\alpha_{2 \mathrm{~A}} \mathrm{AR}$ inhibitor. $\mathrm{A} \beta_{\mathrm{O}}$ increased tau hyperphosphorylation, which both lithium and idazoxan inhibited, leading to the conclusion that both GSK $3 \beta$ and $\alpha_{2 \mathrm{~A}} \mathrm{AR}$ are required to induce potentially cytotoxic tau hyperphosphorylation, with $\alpha_{2 \mathrm{~A}} \mathrm{AR}$ providing a critical connection.

Zhang et al. hypothesized that inhibiting $\alpha_{2 \mathrm{~A}} \mathrm{AR}$ would have therapeutic potential. They treated AD mice carrying APP and PS1 mutation as well as the other transgenic strain previously mentioned at 7.5-8 months of age for 8 weeks with idazoxan to reduce $\alpha_{2 \mathrm{~A}} \mathrm{AR}$ activity. They observed less accumulation of $\mathrm{A} \beta$, microglial activation demonstrating that idazoxan slowed progression of AD. Moreover, they observed decreased tau hyperphosphorylation. These histological changes correlated with improvements to cognitive function, in the Morris water maze, in which the time mice take to escape from a pool of water onto a hidden platform improves with training. Idazoxan-treated mice responded similarly to wild-type mice after several rounds of training indicating substantial improvement over untreated AD mutant mice. Zhang et al. concluded that inhibiting NE signaling through $\alpha_{2 \mathrm{~A}} \mathrm{AR}$ is a potentially effective way to decouple the pathological effects of amyloid and tau in AD.

\section{Stress-Induced SNS Signaling Through NE in Hair Follicles Causes Pigment Loss in Hairs Through Stem Cell Depletion}

Zhang et al. recently reported a mechanism by which stress causes premature graying of hair in mice by a mechanism involving stem cell depletion. ${ }^{30}$ It has been long known that various kinds of stress promote graying of hair in mice and people. In mice, stress is induced by chronic restraints, unpredictably timed stimuli such as loud sounds, or injection of resiniferatoxin (RTX), an analogue of capsaicin. Capsaicin is found in hot peppers.

Zhang et al. subjected mice to 3-5 rounds of stressors and found that hair pigmentation was lost. Plasma levels of corticosterone (human cortisol equivalent) and NE (noradrenaline) increased as would be expected. What was the mechanism?

There are several obvious possibilities: (1) decreased melanin synthesis in the melanocytes that color hair in the follicle; (2) loss of differentiated melanocytes; (3) loss of or problems with the maintenance of melanocyte stem cells (MeSCs). Zhang et al. found that MeSCs were completely lost from the bulge in which they are located in the hair follicle after 5 days, although melanocytes themselves are not affected. After several rounds of hair follicle telogen (rest), anagen (hair growth), and catagen (during which melanocytes die), many follicles were bereft of melanocytes and MeSCs resulting in gray hair.

Using Rag1 mutant mice that lack B and T cells, Zhang et al. still observed graying of hair after stress, ruling out an immune mechanism. Using a mouse strain in which the glucocorticoid receptor (GR) could be genetically excised conditionally with tamoxifen, Zhang et al. observed that corticosterone was not involved as the mice still went gray in response to their stressor regimen. However, depleting ADRB2, which encodes the beta2 adrenergic receptor ( $\beta 2$ adrenoreceptor) using similar conditional mice to that used for the GR experiments, protected the mice from stress-associated gray hair induction.

Signaling studies on wild-type mice suggested that NE induces phosphorylation of cAMP response element-binding protein (CREB) in MeSCs, which could be consistent with later stem cell differentiation, but first other mechanisms had to be ruled out. Assays for apoptosis or necrosis showed no evidence of MeSC death after the mice were stressed. DNA damage is known to induce MeSC differentiation, but an increase in gamma-H2AX that associates with double-stranded DNA was not observed. A more common mechanism for loss of stem cells is overstimulation and/or loss of quiescence. Fifty percent of MeSCs become positive for a marker of cell cycle traversal within 24 hours of RTX injection. At the same time, no effects on cell proliferation or death of mature melanocytes were observed.

Using a transgenic strain in which MeSCs are labeled with green flourescent protein (GFP), Zhang et al. observed a transient increase in GFP positive cells followed by appearance of dendritic branching and moving out of the stem cell niche in the bulge of the hair follicle, suggesting that they differentiated into melanocytes. As might be expected there was an increase in key G1 to $\mathrm{S}$ transition promoter cyclin dependent kinase 2 (CDK) as well as melanocyte specific genes. Importantly injection of NE could induce the same changes in gene expression and phenotype as induced by RTX or other stressors. The key experiments to prove their hypothesis involved blocking cell proliferation by CDK inhibitors flavopiridol or AT7519 and by using a transgenic mouse capable of inducing p27. These proliferation inhibitors all prevented MeSC differentiation and preserved hair pigmentation.

The authors conclude that loss of quiescence drives the stress-mediated depletion of MeSCs and that suppressing MeSC proliferation is sufficient to prevent their loss.

Another key issue is the NE surge that triggers MeSC proliferation and differentiation mediated through the plasma or locally through NE secreting neurons. To rule out plasmaderived NE, the adrenal glands of the mice were removed. However, stressors still induced loss of pigmentation suggesting that the SNS was the origin of NE. Interestingly sympathetic neurons terminate close to the follicle bulge where the MeSCs are located. Rapid induction of $c$-fos, a biomarker for neuronal activity, was observed after RTX injection. Ablating sympathetic nerves with six-hydroxy dopamine, a selective neurotoxin for sympathetic nerves or treating with guanethidine, a drug that blocks NE release from sympathetic neurons, prevented hair graying and MeSC loss. Together these studies suggest that the SNS activation through secretion of NE drives differentiation loss of MeSC to cause stress-mediated loss of hair pigmentation.

Essentially, what Zhang et al. observed is asymmetrical differentiation of a stem cell population where instead of symmetrical cell divisions resulting in one SC and one differentiated cell, all of the SC differentiate. That small changes in the SC microenvironment can so disturb SC maintenance suggests that similar events could be involved in the loss of $\mathrm{SC}$ with aging. This is in addition to mechanisms by which SC lose their ability to differentiate properly as has been reported for sperm, muscle, and hematopoietic stem cells. ${ }^{31}$ 


\section{Medical Implications}

The medical implications of inhibiting $\alpha_{2 \mathrm{~A}} \mathrm{AR}$ in $\mathrm{AD}$ are profound, as a potential therapeutic drug, idazoxan is an investigational drug that has been tested in clinical trials. Decoupling beta-amyloid deposition from hyperphosphorylated tau formation could be of great significance. This needs to be tempered by the complicated phenotype of $\mathrm{AD}$, which may involve prion-like tau ${ }^{32-34}$ or tau-induced cell senescence ${ }^{35}$ among other mechanisms. Once prion-like tau is formed, or senescence is induced, it may take more than blocking an amyloid-tau positive feedback to stop progression. Furthermore, even if AD progression is stopped, there would be a need to reconstitute lost neural circuits.

One of the most interesting aspects of Zhang's results is that it could help explain why immunotherapies targeting $\mathrm{A} \beta$ have mostly failed. ${ }^{36}$ If only nanomolar concentrations of $\mathrm{A} \beta_{\mathrm{O}}$ are sufficient to induce tau hyperphosphorylation and pathology, then antibody clearance strategies are probably doomed to fail as they are unlikely to remove sufficient pathological $\mathrm{A} \beta$.

That SNS signaling through NE can drive loss of hair pigmentation through a classic stem cell loss mechanism invites the question whether a similar mechanism is involved in graying of hair during aging. It is known that loss of catalase plays a key role in hair graying, which could easily be associated with loss of MeSC and melanocytes given associated downregulation of melanocyte biomarkers. ${ }^{37}$ This should be investigated in more depth. Loss of SC may seem difficult to repair, but with the advent of cell reprogramming strategies, ${ }^{38}$ eventually it may become possible to replace or rejuvenate lost SC populations throughout the body. ${ }^{39}$

Finally, given the recently uncovered roles in pathologies for SNS signaling in general and NE-mediated signaling in particular, it would not be surprising to find activation of the SNS playing key roles in numerous examples of physiological dysfunction associated with aging, and studies to uncover such mechanisms should be initiated, especially given the possibility of altering this well-studied component of the ANS with small molecule therapeutics. Moreover, the hypothesized connections of increased SNS activation with cell senescence need to be established.

\section{Author Disclosure Statement}

No competing financial interests exist.

\section{Funding Information}

No funding was received for this article.

\section{References}

1. Balasubramanian P, Hall D, Subramanian M. Sympathetic nervous system as a target for aging and obesity-related cardiovascular diseases. Geroscience 2018;41:13-24.

2. Guyenet PG. The sympathetic control of blood pressure. Nat Rev Neurosci 2006;7:335-346.

3. Lambert E, Straznicky N, Schlaich M, et al. Differing pattern of sympathoexcitation in normal-weight and obesity-related hypertension. Hypertens 2007;50:862-868.

4. Grassi G, Quarti-Trevano F, Seravalle G, et al. Early sympathetic activation in the initial clinical stages of chronic renal failure. Hypertens 2011;57:846-851.
5. Hijmering ML, Stroes ESG, Olijhoek J, Hutten BA, Blankestijn PJ, Rabelink TJ. Sympathetic activation markedly reduces endothelium-dependent, flow-mediated vasodilation. J Am Coll Cardiol 2002;39:683-688.

6. de Souza SBC, Rocha JA, Cuoco MAR, et al. High muscle sympathetic nerve activity is associated with left ventricular dysfunction in treated hypertensive patients. Am J Hypertens 2013;26:912-917.

7. Dinenno FA, Jones PP, Seals DR, Tanaka H. Ageassociated arterial wall thickening is related to elevations in sympathetic activity in healthy humans. Am J Physiol Heart Circ Physiol 2000;278:H1205-H1210.

8. Moreira MC, Pinto IS, Mourão AA, et al. Does the sympathetic nervous system contribute to the pathophysiology of metabolic syndrome? Front Physiol 2015;6:234.

9. Tahara Y, Takatsu Y, Shiraishi T, et al. Age-related circadian disorganization caused by sympathetic dysfunction in peripheral clock regulation. NPJ Aging Mech Dis 2017; $3: 1-11$.

10. Shi Y-C, Lau J, Lin Z, et al. Arcuate NPY controls sympathetic output and BAT function via a relay of tyrosine hydroxylase neurons in the PVN. Cell Metab 2013;17:236248.

11. Kim K, Choe HK. Role of hypothalamus in aging and its underlying cellular mechanisms. Mech Ageing Dev 2019; 177:74-79.

12. Rowe JW, Troen BR. Sympathetic nervous system and aging in man. Endocr Rev 1980;1:167-179.

13. Milne B, Hong M. Increasing longevity by decreasing sympathetic stress-early beta receptor blockade pharmacotherapy. Med Hypotheses 2004;62:755-758.

14. Spindler SR, Mote PL, Li R, Dhahbi JM, Yamakawa A, Flegal JM, et al. $\beta 1$-Adrenergic receptor blockade extends the life span of Drosophila and long-lived mice. Age 2013; 35:2099-2109.

15. Levine HJ. Rest heart rate and life expectancy. J Am Coll Cardiol 1997;30:1104-1106.

16. Stessman J, Jacobs JM, Stessman-Lande I, Gilon D, Leibowitz D. Aging, resting pulse rate, and longevity. J Am Geriatr Soc 2013;61:40-45.

17. Böhm M, Swedberg K, Komajda M, Borer JS, Ford I, Dubost-Brama A, et al. Heart rate as a risk factor in chronic heart failure (SHIFT): the association between heart rate and outcomes in a randomised placebo-controlled trial. Lancet (London, England) 2010;376:886-894.

18. Fox K, Borer JS, Camm AJ, Danchin N, Ferrari R, Lopez Sendon JL, et al. Resting heart rate in cardiovascular disease. J Am Coll Cardiol 2007;50:823-830.

19. Cooney MT, Vartiainen E, Laatikainen T, Laakitainen T, Juolevi A, Dudina A, et al. Elevated resting heart rate is an independent risk factor for cardiovascular disease in healthy men and women. Am Heart J 2010;159:612-619.e3.

20. Nanchen D, Leening MJG, Locatelli I, Cornuz J, Kors JA, Heeringa J, et al. Resting heart rate and the risk of heart failure in healthy adults: the Rotterdam Study. Circulation Heart Failure 2013;6:403-410.

21. Custodis F, Schirmer SH, Baumhäkel M, Heusch G, Böhm M, Laufs U. Vascular Pathophysiology in Response to Increased Heart Rate. J Am Coll Cardiol 2010; 56:1973-1983.

22. Heusch G. Heart rate in the pathophysiology of coronary blood flow and myocardial ischaemia: benefit from selective bradycardic agents. Br J Pharmacol 2008;153: 1589-1601. 
23. Eppinga RN, Hagemeijer Y, Burgess S, Hinds DA, Stefansson K, Gudbjartsson DF, et al. Identification of genomic loci associated with resting heart rate and shared genetic predictors with all-cause mortality. Nat Genet 2016;48: 1557-1563.

24. Koruth JS, Lala A, Pinney S, Reddy VY, Dukkipati SR. The Clinical Use of Ivabradine. J Am Coll Cardiol 2017;70: 1777-1784.

25. Custodis F, Baumhäkel M, Schlimmer N, List F, Gensch C, Böhm M, et al. Heart rate reduction by ivabradine reduces oxidative stress, improves endothelial function, and prevents atherosclerosis in apolipoprotein E-deficient mice. Circulation 2008;117:2377-2387.

26. Gent $S$, Kleinbongard $P$, Dammann $P$, Neuhäuser $M$, Heusch G. Heart rate reduction and longevity in mice. Basic Res Cardiol 2015;110:2

27. Corrada MM, Brookmeyer R, Paganini-Hill A, Berlau D, Kawas $\mathrm{CH}$. Dementia incidence continues to increase with age in the oldest old the 90+ study. Ann Neurol 2010;67:114-121.

28. Huber CM, Yee C, May T, Dhanala A, Mitchell CS. Cognitive decline in preclinical Alzheimer's disease: Amyloidbeta versus tauopathy. J Alzheimers Dis 2018;61:265-281.

29. Zhang $\mathrm{F}$, Gannon $\mathrm{M}$, Chen $\mathrm{Y}$, et al. $\beta$-amyloid redirects norepinephrine signaling to activate the pathogenic GSK3 $\beta /$ tau cascade. Sci Transl Med 2020;12:eaay6931.

30. Zhang B, Ma S, Rachmin I, et al. Hyperactivation of sympathetic nerves drives depletion of melanocyte stem cells. Nature 2020 [Epub ahead of print]; DOI: 10.1038/s41586020-1935-3.

31. Kosan C, Heidel FH, Godmann M, Bierhoff H. Epigenetic erosion in adult stem cells: Drivers and passengers of aging. Cells 2018;7:E237.
32. Clavaguera F, Duyckaerts C, Haik S. Prion-like properties of Tau assemblies. Curr Opin Neurobiol 2020;61:49-57.

33. Ayers JI, Giasson BI, Borchelt DR. Prion-like Spreading in Tauopathies. Biol Psychiatry 2018;83:337-346.

34. Aoyagi A, Condello C, Stöhr J, et al. A $\beta$ and tau prion-like activities decline with longevity in the Alzheimer's disease human brain. Sci Transl Med 2019;11:eaat8462.

35. Musi N, Valentine JM, Sickora KR, et al. Tau protein aggregation is associated with cellular senescence in the brain. Aging Cell 2018;17:e12840.

36. Panza F, Lozupone M, Logroscino G, Imbimbo BP. A critical appraisal of amyloid- $\beta$-targeting therapies for Alzheimer disease. Nat Rev Neurol 2019;15:73-88.

37. Shi Y, Luo L-F, Liu X-M, Zhou Q, Xu S-Z, Lei T-C. Premature graying as a consequence of compromised antioxidant activity in hair bulb melanocytes and their precursors. PLoS One 2014;9:e93589.

38. Liu G, David BT, Trawczynski M, Fessler RG. Advances in pluripotent stem cells: History, mechanisms, technologies, and applications. Stem Cell Rev Rep 2019 [Epub ahead of print]; DOI: 10.1007/s12015-019-09935-x.

39. Wang M, Chen J, Chen F, et al. Rejuvenating strategies of tissue-specific stem cells for healthy aging. Aging Dis 2019;10:871-882.

Address correspondence to: Andrew R. Mendelsohn Regenerative Sciences Institute 1230 Bordeaux Drive Sunnyvale, CA 94089

E-mail: amend@regensci.org 